\title{
Synthesis of spherical microscale and nanoscale modified silica particles using spray-drying and spray-pyrolysis process
}

\author{
C. F. N. Gonin' \\ M. A. C. Berton' \\ M. A. de Goes' \\ L. da Conceição'
}

\section{Abstract}

In the present work, we prepared modified and unmodified microscale and nanoscale silica particles using spray-drying and the spray-pyrolysis process, respectively, which could be an alternative for the production of this kind of product in industrial scale. For it, modified Stöber method was used to synthetize the precursor solution including tetraethyl orthosilicate (TEOS) and triethoxy(octyl)silane (ETOS) as precursors and 3-(Trimethoxysilyl)propyl methacrylate $(\gamma$-MPS) as template for the functionalization. The silica sol passed into both equipment to obtain the final powder. The surface morphology was investigated with Scanning Electron Microscopy (SEM), and the Fourier Transform Infrared spectroscopy (FT-IR) was used to describe the functionalization of the final powder. SEM results show that we successfully synthetized spherical microscale and nanoscale particles with spray-drying and spray-pyrolysis process, respectively. The FT-IR analysis show the functionalization of the silica powder obtained with both technologies. It is possible to consider these technologies as a simple way to produce modified spherical silica particles in an industrial scale.

Keywords: Spray-drying, Spray-pyrolysis, silica, powder.

1 SENAI Institute of Innovation in Electrochemistry, Curitiba, Paraná, Brazil. 


\section{Introduction}

In the recent years, nanomaterials have attracted a lot of attention to develop new products for a wide range of industries [1-2]. The control of the morphology and the surface morphology of those nanomaterials is very important to obtain products with the same chemical and physical properties [3]. That's why, many process were developed to allow the fabrication of such products in an industrial scale. Spray-drying and spray-pyrolysis processes are part of these technologies [3].

Spray-Drying process is widely used for food, chemical, pharmaceutical and materials industries to synthesize microscale particles [1, 3-4]. It consists in solvent evaporation spraying atomized droplets into a high temperature chamber to produce dry powder with controlled size. The main drawback of this method was the polydispersity droplets obtained with conventional spray-drying which can be eliminated by the use of a two fluid nozzle which produce monodispersed droplets [5]. Spray-Pyrolysis technique is widely used to synthesize dense solid such as ceramics [6-7]. This process consists of ultrasonic nebulization of the precursor solution to produce droplets that will be carried into a high temperature furnace by the vector gas to form nanoparticles. Then produced particles with a defined charge are collected by an electrode with an opposite charge and a controlled tension applied.

In the present work, we synthesized modified and unmodified silica microscale and nanoscale particles using the spray-drying and the spray-pyrolysis process, respectively, which could be an alternative for the production scale of this kind of product.

One of well-established procedure to synthetize colloidal silica nanoparticles is the Stöber process [3, 8-11]. It is a sol-gel process where alkoxysilane, as a precursor, is hydrolyzed and condensed in an acidified or basified solvent including a mixture of alcohol and water. The particle size depends of the alkoxysilane type used and the chain length of the alcohol [12]. That's why, tetraethyl orthosilicate (TEOS) as precursor and ethanol (EtOH) or methanol as alcohol solvent are commonly used because they allow the synthesis of the smallest particles. In this work, we used a modified Stöber process to synthesize acrylate modified silica nanoparticles. Z. Luo \& $\mathrm{Al}[13]$ show that it is possible to modify the silica nanoparticle surface using a one-step method. The authors added the surfactant 30 minutes after the beginning of the reaction, when the first silica particles appear. It results to the functionalization of the nanoparticles surface. This procedure was validated by E. Effati \& Al [14] to produce 
acrylate modified but also vinyl modified silica nanoparticles. We used the same procedure for the synthesis except that the final powder will be obtained by spray-drying or spray-pyrolysis process.

\section{Materials and methods}

\subsection{Materials}

Tetraethyl orthosilicate (TEOS, reagent grade, 98\%), triethoxy(octyl)silane (ETOS, $\geq 97,5 \%)$, 3-(Trimethoxysilyl)propyl methacrylate $(\gamma$-MPS, 98\%) and Ammonium Hydroxide $\left(\mathrm{NH}_{4} \mathrm{OH}, 24-26 \%\right)$ were purchased from Sigma-Aldrich and used without further purification. Ethanol Absolute (EtOH, PA, 99.8\%) were obtained from Alphatec. The ultrapure water $\left(\mathrm{H}_{2} \mathrm{O}\right)$ were made in our laboratory using a water purification system Direct-Q 5 UV from EMD Millipore.

\subsection{Synthesis of silica sol using a modified Stöber process}

Firstly, a solution including $700 \mathrm{~mL}$ of EtOH, $85 \mathrm{~mL}$ of $\mathrm{H}_{2} \mathrm{O}$ and 22,4 mL of $\mathrm{NH}_{4} \mathrm{OH}$ was prepared in a $1 \mathrm{~L}$ erlenmeyer. In parallel, a silica precursor solution containing 3,22 $\mathrm{mL}$ of ETOS and $12,81 \mathrm{~mL}$ of TEOS in $20 \mathrm{~mL}$ of EtOH was realized and leaving in low stirring for 10 minutes. This precursor solution was added dropwise in presence of a high speed agitation. After 30 minutes of vigorous stirring, 1,68 mL of $\gamma$-MPS are incorporated for surface modification of silica particles and the reaction was left reacting overnight at room temperature. The silica sol was passed in spray-drying and spray-pyrolysis process to obtain a powder which will be able to be used for the desired application. Unmodified silica particles were also synthetized by the same process without the addition of the surfactant.

\subsection{Synthesis of spherical microscale modified silicate powder via spray- drying}

The silica sol was dried using a Buchi mini spray drier B-290 with a two-fluid nozzle and the air as gas vector. It consists in solvent evaporation spraying atomized droplets into a high temperature chamber to obtain a dry powder with a narrow microparticles sizes distribution as shown in the Figure 1. 


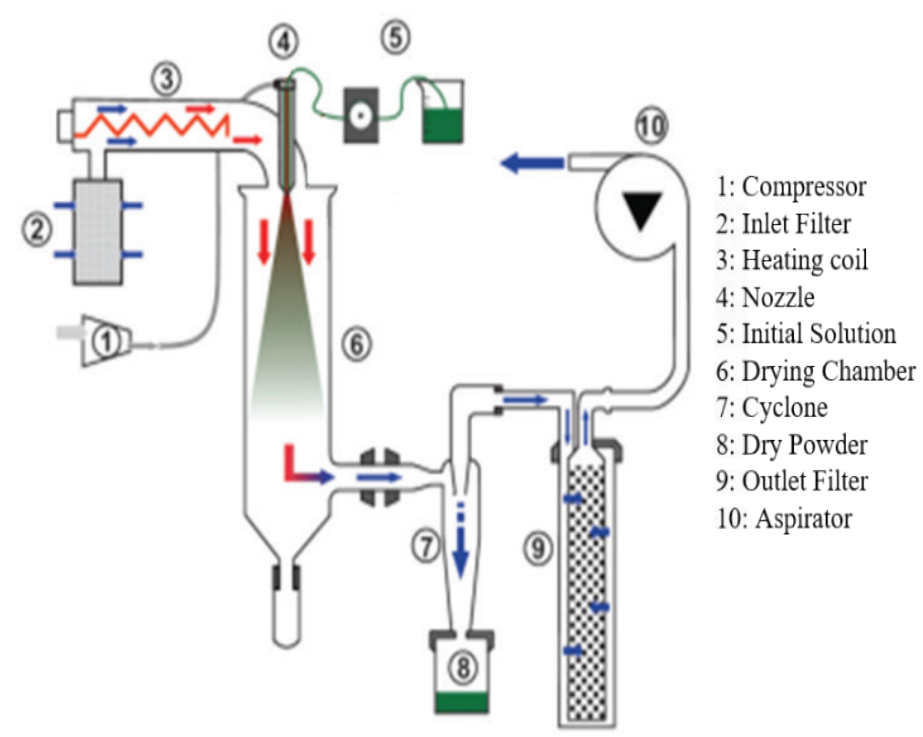

Figure 1 Principle of the Buchi mini spray dryer B-290 equipment

Source: adapted from O. Kaspar \& Al, 2013, p. 526 [15]

At the nozzle, the fluid which is compounded of the precursor solution and the hot air is atomized in the heat chamber for the evaporation step. The controlled parameters are listed in the Table 1. The inlet temperature was controlled at $150{ }^{\circ} \mathrm{C}$. This temperature was chosen to impede the remove of the organic group of the surfactant present at the surface of the silica particles which can happen with higher temperature. The feed rate had kept at $1 \%$, the aspirator rate at $65 \%$ and the gas flow rate at $50 \mathrm{~L} / \mathrm{h}$.

Table 1 Instrument parameters for the spray drying synthesis of the microscale functionalized silica particles.

\begin{tabular}{|l|l|}
\hline \multicolumn{1}{|c|}{ Parameters } & \multicolumn{1}{c|}{ Instrument setting } \\
\hline Aspirator & $65 \%$ \\
\hline Flow rate & $1 \%$ \\
\hline Inlet temperature & $150^{\circ} \mathrm{C}$ \\
\hline Gas flow rate & $50 \mathrm{~L} / \mathrm{h}$ \\
\hline
\end{tabular}

The outlet temperature is influenced by various parameters like the inlet temperature, solid concentration, the solvent used and the spray gas flow. It was measured at about $50^{\circ} \mathrm{C}$. Thanks to the capillarity forces [16], colloidal 
particles are dragged towards each other's to form the final product which will be collected by a collector after passing from the cyclone which allow its separation to the gas. The powder is stored into desiccator at room temperature before being analyzed.

\subsection{Synthesis of spherical nanoscale modified silicate powder via spray- pyrolysis}

Nanoscale powder of silica particles were synthetized using the ultrasonic spray-pyrolysis process. The apparatus used is shown schematically in the Figure 2.

Figure 2 Principle of the spray-pyrolysis process using a Tubular Furnace Fortelab model FII200/HE

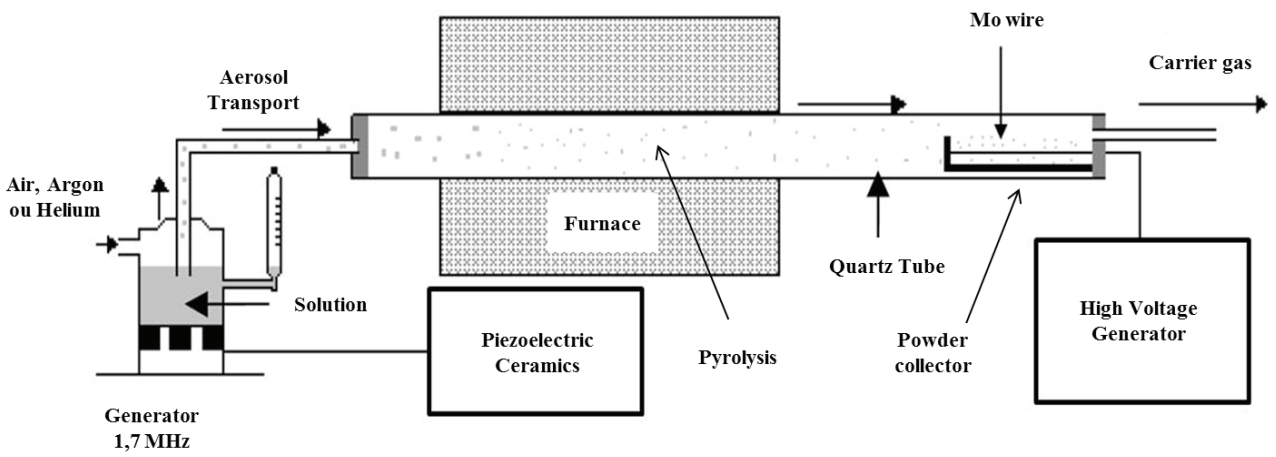

Source: adapted from S. Boulfrad thesis defended in 2007 [17]

As can be seen from the Figure 2, the process consists on the pyrolysis of the silica sol by an ultrasonic nebulizer with a high frequency ultrasonic generator $(1.3 \mathrm{MHz})$. The atomized droplets are carried by a vector gas through a quartz tube. In our case, the gas is the air and is produced by a pressurized air device. The precursor silica sol passed into a tubular furnace model FT1200/ HE purchased from Fortelab where the solvent will be evaporated and the particles decomposed in oxides. For the same reason of the spray drying process, the furnace temperature was controlled at $150^{\circ} \mathrm{C}$. The silica powder is collected electrostatically thanks to a stainless steel electrode and a molybdenum wire polarized using a high voltage generator. The final powder is stored into a desiccator at room temperature before further analysis. All the controlled parameters are shown in the Table 2. 
Table 2 Instrument parameters for the spray drying synthesis of the microscale functionalized silica particles.

\begin{tabular}{|l|l|}
\hline \multicolumn{1}{|c|}{ Parameters } & \multicolumn{1}{c|}{ Instrument setting } \\
\hline Furnace temperature & $150^{\circ} \mathrm{C}$ \\
\hline Ultrasonic Generator Frequency & $1.3 \mathrm{MHz}$ \\
\hline High Voltage Generator & $4000 \mathrm{~V}$ \\
\hline Gas flow rate & $6 \mathrm{~L} / \mathrm{min}$ \\
\hline
\end{tabular}

\subsection{Characterization}

Fourier transform infrared spectroscopy (FT-IR, Bruker HTS-XT) was used to investigate the particles functionalization. The morphology and surface texture of the silica particles were observed by field gun emission scanning electron microscopy (FEG-SEM, Carl Zeiss SUPRA 55VP). Particles size distribution was analyzed by laser diffraction spectrometry (LDS) using a Microtrac S3500 laser diffraction analyzer with sample delivery controller accessory.

\section{Results and discussion}

\subsection{FT-IR results}

The presence of the functionalization by the $\gamma$-MPS was confirmed by FTIR. The FT-IR results of unmodified (Figure 3.c) and modified silica particles obtained by spray-drying (Figure 3.a) and spray-pyrolysis (Figure 3.b) are shown in the Figure 3. The absorption peaks between $1100 \mathrm{~cm}-1$ and $475 \mathrm{~cm}-1$ are characteristic of the silica particle according to the literature [13-14, 18-19]. Indeed, peaks near $1100 \mathrm{~cm}-1,790 \mathrm{~cm}-1$ and $450 \mathrm{~cm}-1$ are ascribed respectively to the asymmetric stretching mode, the bending vibration and rocking vibration absorption of Si-O-Si bond which is characteristic of the condensation of silicon alkoxyde. The absorption peaks near $940 \mathrm{~cm}-1$ belong to the Si-O stretching vibration of the Si-OH bond. Peaks near $3350 \mathrm{~cm}-1$ and $1630 \mathrm{~cm}-1$ are characteristic of the $\mathrm{H}-\mathrm{O}-\mathrm{H}$ stretching and bending mode of the residual water respectively. Peaks at $1700 \mathrm{~cm}-1$ and $2925 \mathrm{~cm}-1$ are attributed to the functional group $\mathrm{C}=\mathrm{O}$ and the stretching vibration of $-\mathrm{CH} 2$ respectively which could confirm the presence of organic functional group at the surface of the modified silica particles. Thus, it could be proved the functionalization of silica particle is successfully achieved. 
Synthesis of spherical microscale and nanoscale modified silica particles...
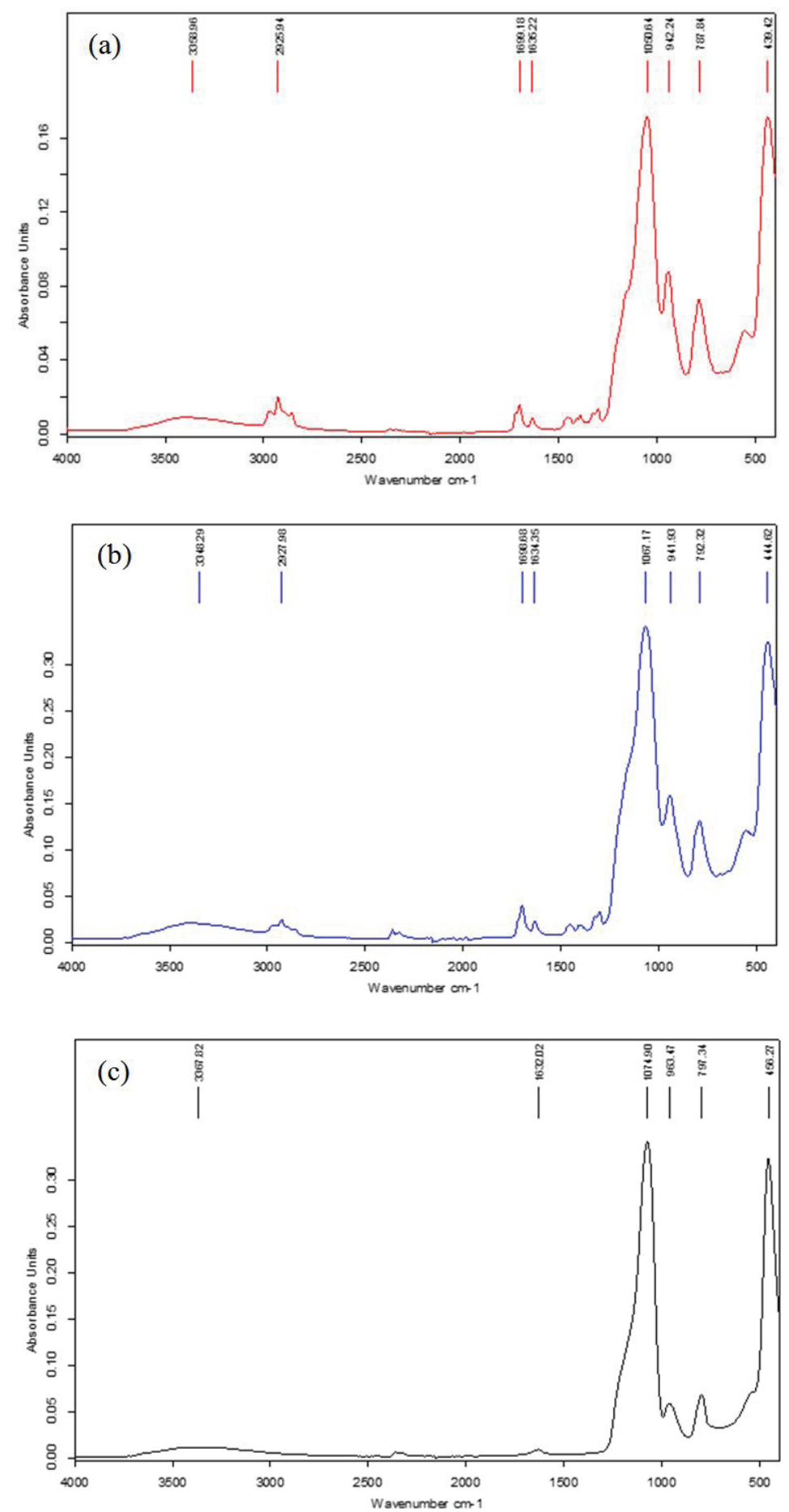

Figure 3 FT-IR results of modified silica powder obtained by (a) spray-drying and (b) spray-pyrolysis and (c) unmodified silica particles 


\subsection{FEG-SEM results}

The morphology and surface texture of the silica powder obtained by spray-drying and spray-pyrolysis process were investigated by FEG-SEM. Results are described in the Figure 4.
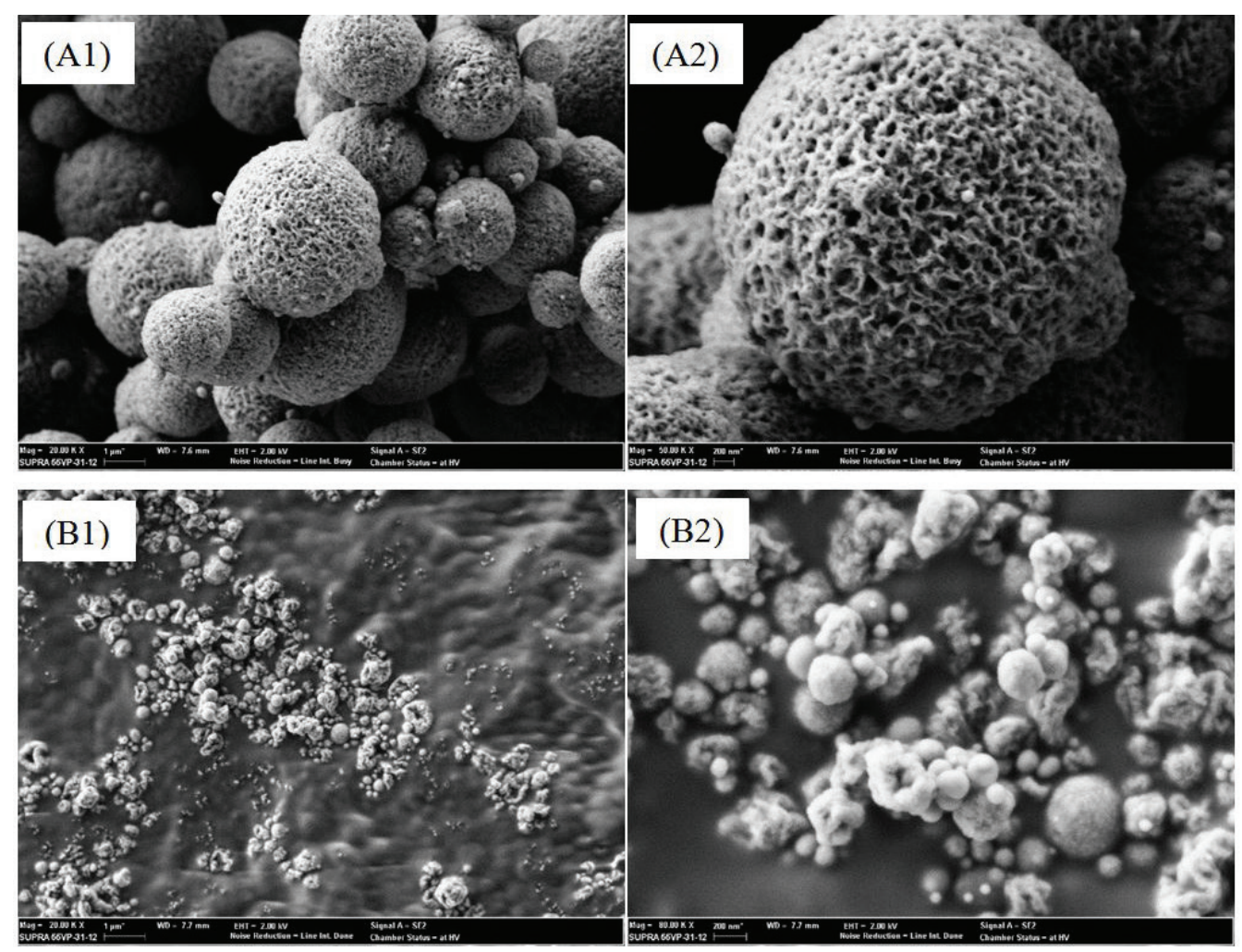

Figure 4 FEG-SEM images of modified silica particles obtained by (AI)/(A2) spray drying and (B1)/(B2) spray pyrolysis.

Modified particles obtained by spray-drying exhibit uniformly spherical morphology (Figure 4. A1) with high surface porosity (Figure 4. A2). It could be explained by the aggregation of the silica nanoparticle in the precursor solution during the spray-drying process.

In the spray pyrolysis case, FEG-SEM images exhibit a spherical structure too. However, the morphology distribution seems to be more dispersed than the microparticles obtained by spray drying. It could be resulted by the ultrasonic atomization behavior of the precursor solution which shown difficulties to form an aerosol. FEG-SEM images also show the smaller particles size obtained with spray pyrolysis process with a nanoscale distribution. 


\subsection{Particle size analysis}

The particle size distribution was investigated by LDS using water as liquid vector and a refractive index of 1.51. Results are exposed in the Figure 5.
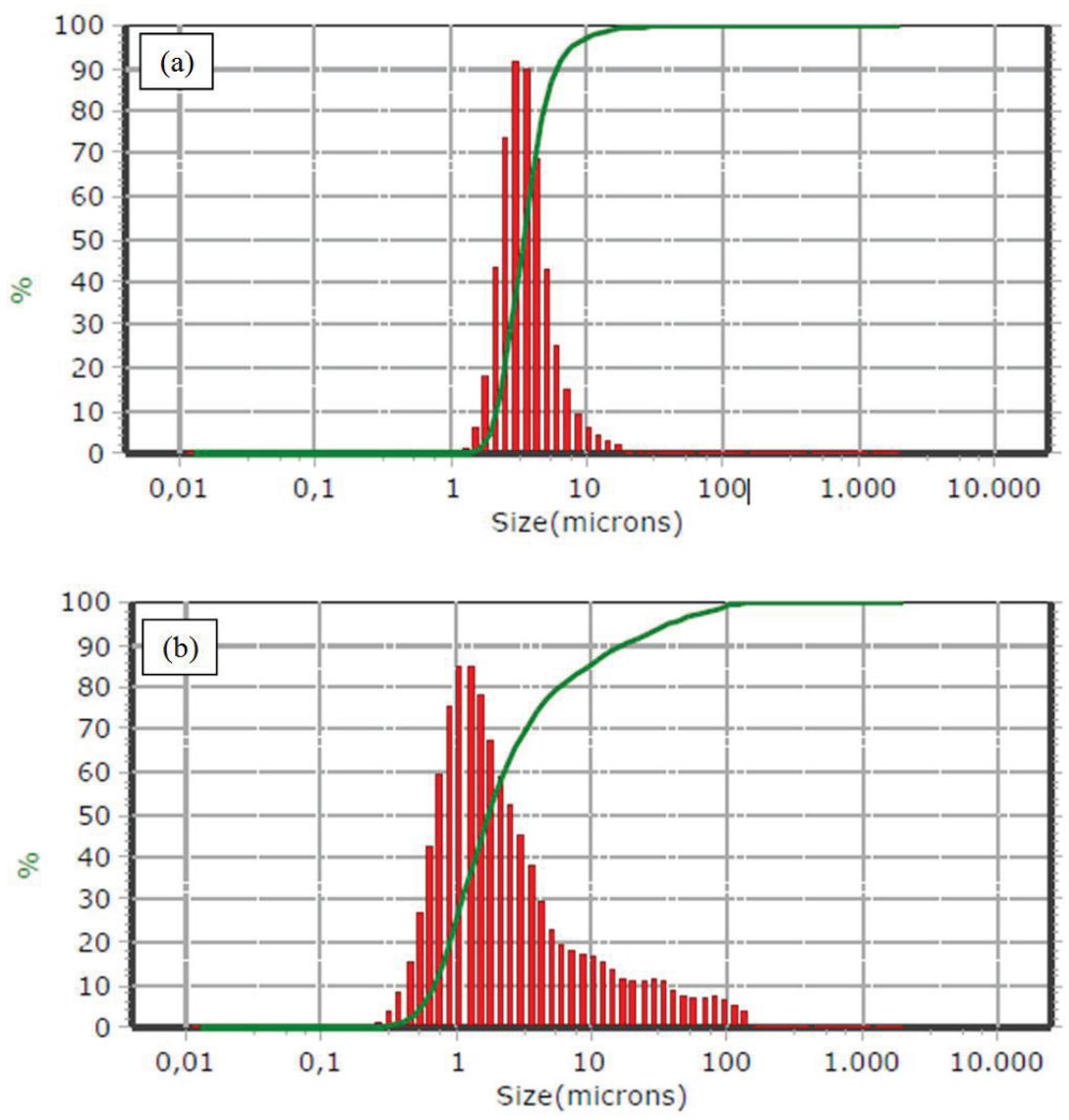

Figure 5 LDS results for acrylate modified silica powder obtained by (a) spray drying and (b) spray pyrolysis.

LDS results show that the medium size is around $3.4 \mu \mathrm{m}$ for the spray drying process (Figure 5. a) which is in agreement with the FEG-SEM images.

For the spray pyrolysis process, the medium size determined is around 1.7 $\mu \mathrm{m}$ (Figure 5.b). It is also possible to observe a larger particles distribution size for this techniques ranging to nanoscale to microscale size. It remains in line with FEG-SEM images which show nanoscale silica particles and could be due to the difficulties visualized during the atomization process. Thus, it's possible to note that the resulting powder in spray pyrolysis process is forming agglomerates and the size obtained in this analysis could be associated to them. 


\section{Conclusion}

In the present work, we successfully synthesized uniformly spherical microscale acrylate modified silica particles using spray drying with a microscale distribution size. We also showed that, in the case of the spray pyrolysis, modified silica nanopowder could be synthetized with a particle size below of $1 \mu \mathrm{m}$. But the process need to be adapted because of the ultrasonic atomization behavior of the precursor solution. However, results obtained are encouraging and it is possible to imagine using this process in the industrial scale. For both technologies, the presence of the functionalization group in the silica particles surfaces was confirmed by the FT-IR spectroscopy. In the future, it is possible to consider the synthesis of other kind of modified spherical microscale and nanoscale modified silica particles using another template than the $\gamma$-MPS with spray drying and spray pyrolysis process, respectively for a wide range of applications.

\section{Acknowledgment}

The authors thank the funding agency "National Counsel of Technological and Scientific Development" (CNPq) from Brazil (Processes n 351396/2015-2) for the financial support to this project and the SENAI Institute of Innovation in Laser from Brazil for the FEG-SEM analysis.

\section{References}

[1] S. Zellmer, M. Lindenau, S. Michel, G. Garnweitner, C. Schilde, Influence of surface modification on structure formation and micromechanical properties of spray-dried silica aggregates, Journal of Colloid and Interface Science, 2016, vol. 464, 183-190.

[2] H. Gleiter, Nanocrystalline Materials, Progress in Materials Science, 1989, vol. 33, 223-315.

[3] Progress in developing spray-drying methods for the production of controlled morphology particles: From the nanometer to submicrometer size ranges, Journal Advanced Powder Technology, 2011, vol. 22, 1-19.

[4] K. Waldron, W. Duo Wu, Z. Wu, W. Liu, C. Selomulya, D. Zhao, X. Dong Chen, Formation of monodisperse silica microparticles via spray drying, Journal of Colloids and Interface Science, 2014, vol. 418. 
[5] D. Sen, J. Bahadur, Avik Das, S. Mazumder, J.S. Melo, H. Frielinghaus, R. Loidl, E. Coli imprinted nano-stuctured silica micro-granules by spray-drying: Optimization and calcination temperature, Journal Colloids and Surfaces B: Biointerfaces, 2015, vol. 127, 164-171.

[6] H. Ran Jang, H-J. Oh, J-H. Kim, K. Youl Jung, Syntheis of mesoporous spherical sílica via spray pyrolysis: Pore size control ans evaluation of performance in paclitaxel pre-purification, Journal of Microporous and Mesoporous Mateirials, 2013, vol. 165, 219-227.

[7] D. Jong Seo, S. Bin Park, Y. Chan Kang, K. Leong Choy, Formation of ZnO, $\mathrm{MgO}$ and $\mathrm{NiO}$ nanoparticles from aqueous droplets in flame reactor, Journal of Nanoparticle Research, 2003, vol. 5, 199-210.

[8] W. Stöber, A. Fink, E. Bohn, Controlled growth of monodispersed silica spheres in the micron size range, Journal of Colloids and Interface Science, 1968, vol. 26, 62-69.

[9] H. Zou, S. Wu, J. Shen, Polymer/Silicate Nanocomposites: Preparation, Characterization, Properties and Applications, Chemical Reviews, 2008, vol. 108, 3893-3957.

[10] T. Ribeiro, C. Baleizão, J.P.S Farinha, Functional Films from Silica/Polymer Nanoparticles, Journal Materials, 2014, vol. 7, 3881-3900.

[11] T. Golhami, M. Salavati-Niasari, M. Bazarganipour, E. Noori, Synthesis and characterization of spherical silica nanoparticles by modified Stöber process assisted by organic ligand, Journal Superlattices and Microstructures, 2013, vol. $61,33-41$.

[12] K. Sreenivasa Rao, K. El-Hami, T. Kodaki, K. Matsushige, K. Makino, A novel methof for synthesis of silica nanoparticles, 2005, vol. 289, 125-131.

[13] Z. Luo, R.Y. Hong, H.D. Xie, W.G. Feng, One-step synthesis of functional silica nanoparticles for reinforcement of polyurethane coatings, Journal Powder Technology, 2012, vol. 218, 23-30.

[14] E. Effati, B. Pourabbas, One-pot synthesis of sub-50 nm vinyl- and acrylate-modified silica nanparticles, Journal Powder Technology, 2012, vol. 219, 276-283. 
[15] O. Kaspar, V. Tokarova, G. S Nyanhongo, G. Gubitz, F. Stepanek, Effect of cross-linking method on the activity of spray-dried chitosan microparticles with immobilized laccase, Food and bioproducts processing, 2013, vol. 91, p 525-533.

[16] D. Sen, J. Bahadur, A. Das, S. Mazumder, J. S. Melo, H. Frielinghaus, R. Loidl, E. coli imprinted nano-structured silica micro-granules by spray drying: Optimization of calcination temperature, Colloids and Surfaces B: Biointerfaces, 2015, vol. 127, p 164-171.

[17] S. Boulfrad, Influence de la nanostructure sur les propriétés électriques et la stabilité de zircones à l'état massif, $\mathrm{PhD}$ thesis defended the november, $15^{\text {th }}$ 2007 at the Institut National Polytechnique de Grenoble, France.

[18] V. Venkatathri, preparation of silica nanoparticles trough coating with octyldecyltrimethoxy silane, Indian Journal of chemistry, 2007, vol. 46A, 1955-1958.

[19] X. Gao, Y. Zhu, X. Zhao, Z. Wang, D. An, Y. Ma, S. Guan, Y. Du, B. Zhou, Synthesis and characterization of polyurethane/SiO2 nanocomposites, Journal of Applied Surface Science, 2011, vol. 257, 4719-4724. 